\title{
Achados histológicos e sobrevida na fibrose pulmonar idiopática*
}

\author{
H istological features and survival in idiopathic pulmonary fibrosis
}

\author{
Ester nel A parecida Martins Coletta ${ }^{1}$, Carlos Alberto de Castro Pereira ${ }^{2}$ (te sbpt), Rimarcs Gomes Ferreira ${ }^{3}$, \\ Adalberto Sperb Rubin ${ }^{4}$, Lucimara Sonja Villela ${ }^{5}$, Tatiana Malmeiros ${ }^{6}$ (Te SbPt), J oão Norberto Stávale ${ }^{3}$
}

Introdução: A fibrose pulmonar idiopática foi recentemente redefinida como pneumonia intersticial usual de etiologia desconhecida. 0 valor prognóstico dos achados histológicos deve ser reavaliado.

O bjetivo: N este estudo foram correlacionados os achados histológicos e alguns dados clínicos e funcionais (duração dos sintomas, capacidade vital forçada, idade, sexo, hábito de fumar) com a sobrevida.

Método: Foram estudados 51 pacientes portadores de fibrose pulmonar idiopática. A média de idade foi de $66 \pm 8$ anos. Vinte e um pacientes eram do sexo feminino; 26 eram fumantes ou ex-fumantes. Todos apresentavam quadro de pneumonia intersticial usual na histologia. Grau de faveolamento, fibrose estabelecida, descamação, celularidade, espessamento vascular miointimal e focos fibroblásticos foram graduados por método semiquantitativo.

Resultados: A mediana do tempo de sintomas foi de 12 meses e a capacidade vital forçada inicial foi de $72 \pm 21 \%$. Por análise de risco proporcional de Cox, a sobrevida correlacionou-se, de maneira significativa $(p \leq 0,05)$ e inversa, com 0 tempo de história, com a extensão dos focos fibroblásticos e com o espessamento miointimal da parede dos vasos. Focos fibroblásticos esparsos e espessamento miointimal envolvendo menos de $50 \%$ dos vasos foram preditivos de maior sobrevida. Sexo, idade, capacidade vital forçada, grau de inflamação e celularidade não se correlacionaram com a sobrevida.

Conclusão: A análise semiquantitativa da biópsia pulmonar em portadores de fibrose pulmonar idiopática fornece informações prognósticas relevantes. (J Pneumol 2003;29(6):371-8)

Descritores - Fibrose pulmonar. Doenças pulmonares intersticiais. A nálise de sobrevivência.
Background: Idiopathic pulmonary fibrosis was recently redefined as usual interstitial pneumonia of unknown etiology. Consequently, the prognostic value of histological findings needs to be reassessed.

O bjective: To correlate clinical, functional and histological findings with survival in patients with idiopathic pulmonary fibrosis.

Method: Patients ( $n=51$; mean age: $66 \pm 8$ years; gender: 21 females/ 30 males) were evaluated. Of the 51, 26 were smokers or ex-smokers. Duration of symptoms, forced vital capacity and smoking habits were recorded. All patients presented usual interstitial pneumonia verified through histology. Degree of honeycombing, established fibrosis, desquamation, cellularity, myointimal thickening of blood vessels and number of fibroblastic foci were graded according to the semiquantitative method.

Results: Median duration of symptoms was 12 months and initial forced vital capacity was $72 \pm 21 \%$. Cox multivariate analysis revealed that survival correlated inversely and significantly $(p \leq 0.05)$ with duration of symptoms and fibroblastic foci score, as well as with myointimal thickening of blood vessels. Limited numbers of fibroblastic foci, as well as myointimal thickening involving less than $50 \%$ of blood vessels, were predictive of greater survival. No correlation with survival was found for gender, age, forced vital capacity, inflammation or degree of cellularity.

Conclusion: Semiquantitative analysis of lung biopsies yields relevant prognostic information regarding patients with usual interstitial pneumonia.

Key words - Pulmonary fibrosis. Lung diseases, interstitial. Survival analysis.

3. Professor Doutor Adjunto do Departamento de Patologia da Unifesp.

4. Doutor em Pneumologia. Pavilhão Pereira Filho, Santa Casa, Porto A legre, RS.

5. M édica Residente do Departamento de Patologia do HSPE.

6. Pós-graduanda da Disciplina de Pneumologia da Unifesp. Título de especialista pela Sociedade Brasileira de Pneumologia e Tisiologia.

Endereço para correspondência - Rua Botucatu, 740, Departamento de Patologia, Edifício Lemos Torres - 04023-900 - São Paulo, SP.

Recebido para publicação em 31/3/03. Aprovado, após revisão, em 16/9/03. 


\section{$\overline{\text { Veja cometários no Editorial, na página } 335}$}

\section{INTRODUÇÃO}

Diversas classificações foram propostas para as pneumonias intersticiais idiopáticas desde os estudos de Liebow, em 1975.(1-3) Em algumas, ${ }^{(2)}$ o termo fibrose pulmonar idiopática (FPI) foi usado para englobar diversas entidades, porém, em $2000^{(4)}$, foi proposto o uso dessa designação apenas para os casos associados à pneumonia intersticial usual (UIP) na biópsia cirúrgica. Em 1994(5) foi caracterizada a pneumonia intersticial não específica (NSIP) como uma entidade que deveria ser diferenciada da UIP. A validade dessa proposta tem sido confirmada em diversas séries, nas quais se verificou melhor prognóstico dessa doença quando comparada com a UIP..$^{(6-8)}$

O diagnóstico de fibrose pulmonar idiopática é freqüentemente aceito em bases clínicas, porém, para o diagnóstico definitivo, é necessário biópsia cirúrgica. Os achados histológicos considerados essenciais para o diagnóstico incluem um processo intersticial com distribuição temporal heterogênea, com áreas de pulmão preservado ao lado de outras comprometidas por fibrose, remodelamento do parênquima pulmonar e presença de focos fibroblásti$\cos ^{(3,9,10)}$

As doenças intersticiais em geral teriam como seqüência evolutiva uma inflamação inicial (alveolite) com progressão para fibrose irreversível. Com bases nesses achados, foram feitas propostas de quantificação da extensão da inflamação e da fibrose nas biópsias cirúrgicas. ${ }^{(11)} \mathrm{Em}$ $1998^{(3)}$ foi sugerido que a inflamação não é relevante na fibrose pulmonar idiopática e que os focos fibroblásticos seriam a lesão inicial. Em um estudo recente, a extensão do tecido de granulação e a extensão de "tecido conectivo jovem" intersticial relacionaram-se diretamente com a sobrevida, porém, o grau de fibrose, não.(12) Em outro estudo utilizando o mesmo escore anatomopatológico,(11) apenas o escore de fibrose foi preditivo da sobrevida. ${ }^{13)}$ O escore utilizado nos dois estudos inclui os focos fibroblásticos tanto no escore de inflamação ("tecido de granulação" que soma achados em alvéolos, ductos alveolares e bronquíolos) quanto no escore de fibrose, em que aparecem como "tecido conjuntivo jovem na parede alveolar", e são somados a outros achados de fibrose como o faveolamento. Um terceiro estudo, envolvendo 85 pacientes, não encontrou valor prognóstico para os focos fibroblásticos, avaliados isoladamente na pneumonia intersticial usual.(14) No escore de fibrose acima citado,(11) alterações vasculares (espessamento miointimal) e hiperplasia de musculatura lisa foram adicionados, mas a importância destes achados isoladamente é discutível. ${ }^{(12)} \mathrm{A}$ angiogênese aumentada pode promover a fibrogênese na

\author{
Siglas e abreviaturas utilizadas neste trabalho \\ CO - Monóxido de carbono \\ CVF - Capacidade vital forçada \\ FPI - Fibrose pulmonar idiopática \\ NSIP Pneumonia intersticial não específica \\ UIP - Pneumonia intersticial usual
}

FPI. ${ }^{(15)}$ A lém disso, foi sugerido recentemente que a lesão microvascular poderia ser o evento inicial e induzir a fibrose pulmonar. ${ }^{(16)}$ Remodelamento vascular extenso ocorre nas áreas de fibrose pulmonar, o que poderia indicar doença mais avançada e pior prognóstico.

0 objetivo do presente estudo foi determinar o valor prognóstico dos achados histológicos e de outros dados, a saber, duração dos sintomas, capacidade vital forçada $(C V F)$, idade, sexo e hábito de fumar, em relação à sobrevida, em pacientes com fibrose pulmonar idiopática, caracterizada por quadro anatomopatológico de pneumonia intersticial usual.

\section{Método}

Cinqüenta e um pacientes portadores de fibrose pulmonar idiopática comprovada histologicamente por biópsia foram incluídos na análise final. Os achados gerais são mostrados na Tabela 1. Nenhum paciente tinha sido previamente tratado. Os casos eram procedentes da Escola Paulista de Medicina, do Hospital do Servidor Público Estadual de São Paulo e do Pavilhão Pereira Filho, de Porto Alegre. Foram excluídas outras doenças intersticiais após a revisão das lâminas, tais como pneumonia não específica e de hipersensibilidade, e casos de pulmão terminal. Foram excluídos casos associados a doenças do colágeno, com material inadequado e sem dados evolutivos, ou com óbito nos primeiros 30 dias após a biópsia. Casos com etiologia potencial para pneumonia intersti-

TABELA 1

Achados gerais em 51 portadores de fibrose pulmonar idiopática 0 s valores são expressos em média \pm desvio padrão

\begin{tabular}{lc}
\hline \multicolumn{1}{c}{ Achado - gerais } & Valores \\
\hline Idade em anos & $66 \pm 8$ \\
Sexo: feminino/masculino & $21 / 30$ \\
Fumantes ou ex-fumantes/Não fumantes & $26 / 25$ \\
Tempo de sintomas em meses, mediana (Q 1-Q 3) & $12 ;(5-24)$ \\
CVF, percentagem do previsto & $72 \pm 21$ \\
CVF inicial, L & $2,26 \pm 0,64$ \\
\hline
\end{tabular}

$*_{n}=48 ; \mathrm{Q} 1-\mathrm{Q} 3=$ Intervalo interquartilítico entre quartil 25 e quartil 75 . 
cial usual, como pneumonia de hipersensibilidade, foram também desconsiderados, após a revisão dos dados clínicos. A pós o teste de distribuição, três casos com histologia compatível foram excluídos por tempo de sobrevida discrepante (132, 179 e 191 meses). Foram ainda excluídos do is casos com outros achados tidos como discrepantes: baixa idade em um (38 anos) e exuberância desproporcional das lesões vasculares em outro.

Informações sobre os óbitos foram obtidas no serviço de registro e arquivo das instituições e através de consulta ao Programa de Aprimoramento das Informações de Mortalidade no Município de São Paulo (PRO-AIM).

Em 11 casos o local da biópsia não pôde ser determinado retrospectivamente. Nos demais as biópsias foram obtidas isoladamente da língula em 12 casos e nos 28 restantes de outros lobos.

Os fragmentos de pulmão foram analisados pelas técnicas usuais com hematoxilina e eosina (HE) e tricrômico de Masson. As biópsias pulmonares foram revistas por dois patologistas com grande experiência no diagnóstico de doenças intersticiais pulmonares (Ester N ei A parecida Martins e Rimarcs Gomes Ferreira), sem conhecimento dos desfechos de estudo. Foram calculados os valores médios das leituras dos dois observadores, que leram as lâminas de maneira independente, para achados histológicos pelo método semiquantitativo.(11) $Q$ uando houve discordância significativa (mais de dois pontos de diferença em determinado escore), um consenso final foi alcançado. Graduação de 0 a 5 (0 - ausente; 1 - ocasional; 2 menos que $25 \%, 3$ - de 25 a $49 \%, 4$ - de 50 a $75 \%$, e 5 - mais que $75 \%$ ) foi aplicada às seguintes alterações: extensão e intensidade da celularidade na parede alveolar (interstício), extensão e intensidade da celularidade nos espaços alveolares (descamação), tecido conjuntivo "jovem" intersticial em paredes alveolares (focos fibroblásticos), fibrose intersticial estabelecida (incluindo faveolamento), cistos ("favo de mel"), músculo liso metaplásico no interstício (excluído músculo liso associado às vias aéreas e vasos) e espessamento miointimal dos vasos. 0 escore para metaplasia alveolar foi desconsiderado.

O escore final considerado, após a análise de vários campos, foi o predominante, segundo avaliação subjetiva. A avaliação funcional inicial foi feita através da medida da CVF. Os valores previstos foram baseados em dados brasileiros. ${ }^{(17)}$ As curvas de sobrevida foram analisadas em relação à idade, ao sexo, ao tempo de história, ao tabagismo, à CVF e aos achados histológicos. A sobrevida foi avaliada até a data de 31 de julho de 2001.

0 programa estatístico utilizado foi o SPSS versão 10 . A concordância entre os patologistas na análise independente das lâminas foi quantificada usando-se o coeficiente de kappa (estes dados foram anteriormente publicados)(18). A distribuição das variáveis quantitativas foi verificada pelo teste de Kolmogorov-Smirnov. As médias foram comparadas utilizando-se o teste t de Student para as variáveis que não rejeitaram a distribuição normal, ou o teste de Mann-Whitney, para as variáveis cuja normalidade foi rejeitada.

Para investigar a relação entre as variáveis envolvendo o escore anatomopatológico foi utilizado o coeficiente de correlação de Spearman (rho). A associação entre variáveis qualitativas foi verificada através do teste do $\chi^{2}$. Para avaliar a influência das variáveis na sobrevida dos pacientes, foi utilizada a regressão de risco proporcional de Cox. Curvas de Kaplan-M eier foram utilizadas para expressar a sobrevida geral e para variáveis transformadas em categóricas, utilizando-se diversos pontos de corte. Para a comparação das curvas, foi utilizado o teste de log-rank. Foram considerados significativos resultados cujo nível descritivo (valores de $p$ ) foram inferiores a $5 \%$.

\section{Resultados}

A mediana do tempo de acompanhamento foi de 26 meses, variando de três a 103 meses. Os escores histológicos são mostrados na Tabela 2. Em média, os doentes eram idosos e com distúrbio restritivo moderado. Todos os pacientes tinham, necessariamente, focos fibroblásticos na biópsia. Em apenas um caso não se evidenciou faveolamento à microscopia.

Do total de pacientes, 26 eram fumantes ou ex-fumantes. Os fumantes e ex-fumantes apresentaram maior escore histológico de descamação, $5,25 \pm 1,58$, quando comparados com os não fumantes, $4,32 \pm 0,84$ ( $p=$ 0,012 ). Em relação às demais variáveis, incluindo as medidas de CVF, não houve diferenças significantes entre os não fumantes e os demais.

Dos 51 pacientes estudados, 25 (49\%) morreram e 26 estavam vivos ao final do estudo. Todos os óbitos, exceto em um caso, foram atribuídos direta (insuficiência respiratória) ou indiretamente (infecções pulmonares) à fibro-

TABELA 2

Escores histológicos em 51 portadores de fibrose pulmonar idiopática

\begin{tabular}{ll}
\hline \multicolumn{1}{c}{ Escores histológicos } & X \pm D P \\
\hline Fibrose estabelecida, incluindo faveolamento & $3,8 \pm 0,7$ \\
Faveolamento & $2,9 \pm 0,9$ \\
Focos fibroblásticos & $2,1 \pm 0,6$ \\
Descamação & $4,8 \pm 1,3$ \\
Celularidade & $5,9 \pm 1,1$ \\
Hiperplasia músculo liso & $2,5 \pm 0,7$ \\
Espessamento miointimal & $2,9 \pm 0,7$ \\
\hline
\end{tabular}




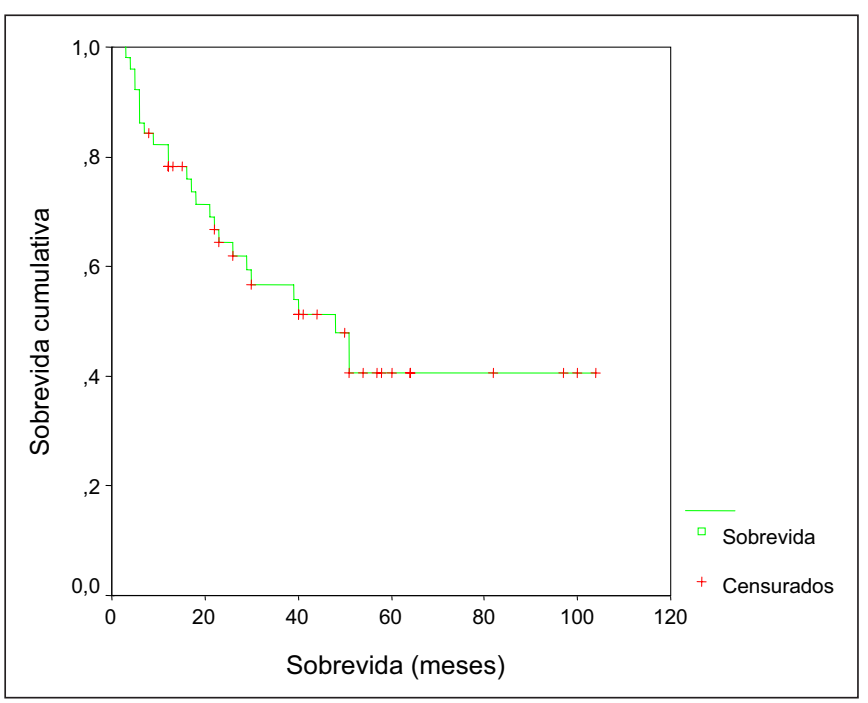

Figura 1 - Sobrevida geral em 51 portadores de fibrose pulmonar idiopática

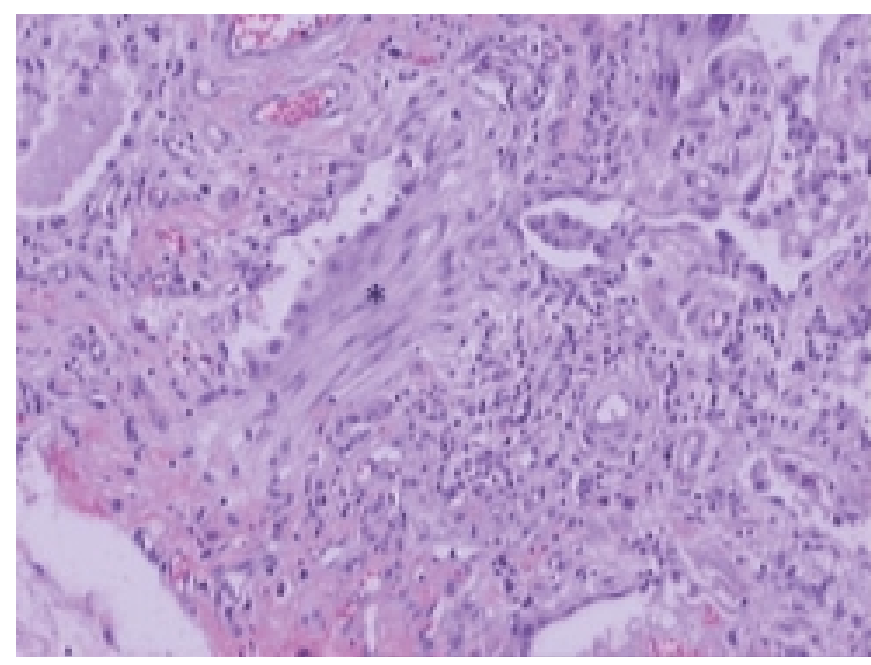

Figura 3 - Foco fibroblástico típico (*)

se. A curva geral de sobrevida, estimada pela curva de Kaplan-Meyer, é mostrada na Figura 1. A mediana geral de sobrevida foi de 48 meses.

Os achados histológicos em relação à sobrevida foram avaliados inicialmente por regressão de Cox para os achados individuais. A penas os escores de focos fibroblásticos e de espessamento miointimal correlacionaram-se significativamente $(p<0,05)$ com a sobrevida. 0 escore de faveolamento e o escore de fibrose estabelecida (que inclui faveolamento) correlacionaram-se marginalmente com a sobrevida ( $p=0,08$ e 0,07 , respectivamente).

A mediana de sobrevida para o grupo com baixo escore $(<2$, focos esparsos) de focos fibroblásticos $(n=14)$ excedeu 70 meses, enquanto que no grupo com escore $\geq$

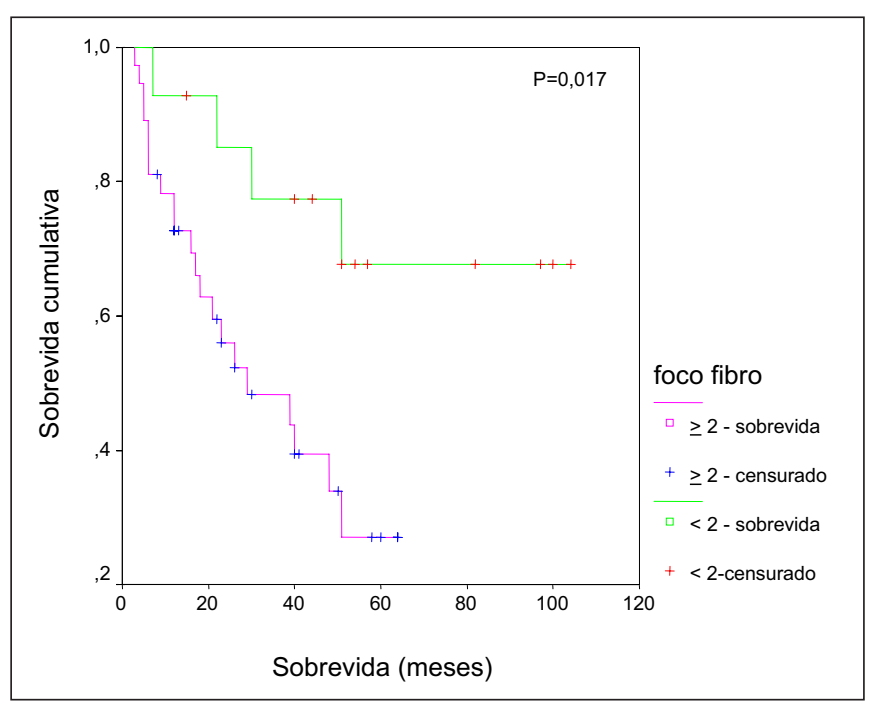

Figura 2 - Sobrevida de pacientes com FPI separados por escore de focos fibroblásticos na biópsia pulmonar
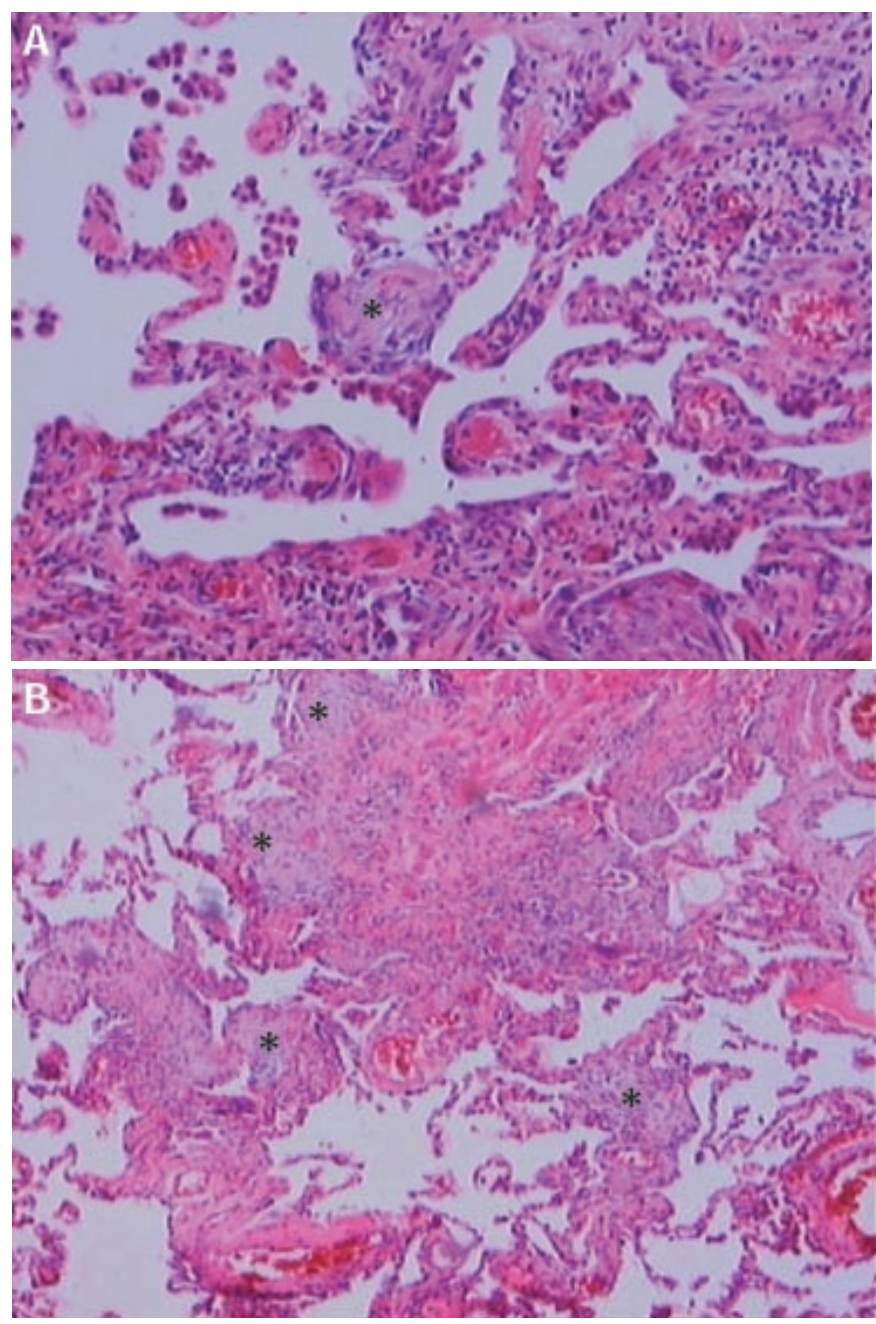

Figura $\mathbf{4 A}$ e $\boldsymbol{B}$ - Focos fibroblásticos esparsos(*) (A) e profusos $(*)$ (B). Aumento de 100x. 
$2(n=37)$ foi de 29 meses (log-rank $=5,72, p=0,017$ ). As curvas de Kaplan-Meyer para esses achados são mostradas na Figura 2. U m foco fibroblástico típico é mostrado na Figura 3. Exemplos de escores de focos fibroblásticos esparsos e profusos representativos são mostrados na Figura 4 A e B. 0 escore de focos fibroblásticos correlacionou-se de maneira direta com a CVF (rho $=0,43, p=$ 0,003).

Para o faveolamento, a melhor separação qualitativa foi obtida dividindo-se os pacientes entre os que apresentavam escore $\leq 3$ ( $50 \%$ ou menos do campo com maior comprometimento na biópsia com faveolamento) e $>3$ (log-rank $=2,98, p=0,084$ ). A pós cinco anos, 38\% dos pacientes com escore de faveolamento $>3$ estavam vivos enquanto que o estavam $72 \%$ no grupo com escore de faveolamento $\leq 3$

No grupo de 37 pacientes com escore de espessamento miointimal $\leq 3$ pontos $(\leq 50 \%$ dos vasos), foram observados 14 óbitos (38\%) e no grupo de 14 com escore acima de três pontos, 11 óbitos $\left(79 \% ; \chi^{2}=6,74, p=\right.$ 0,009 ). A mediana de sobrevida para o grupo com escore $\leq 3$ para o espessamento miointimal excedeu 50 meses e foi de 12 meses no grupo com escore $>3$ (log-rank $=10,86, p=0,001)$. A curva de Kaplan-Meyer para esses achados é mostrada na Figura 5. Exemplos de escores representativos de espessamento miointimal são mostrados na Figura $6 \mathrm{~A}$ e $\mathrm{B}$.

Q uando as quatro variáveis anatomopatológicas (focos fibroblásticos, fibrose estabelecida, faveolamento e espessamento miointimal) que tiveram correlação considerada relevante $(p<0,10)$ foram regredidas contra a sobrevida por um modelo multivariado de Cox, apenas o espessamento miointimal permaneceu significante $(p=0,036)$.

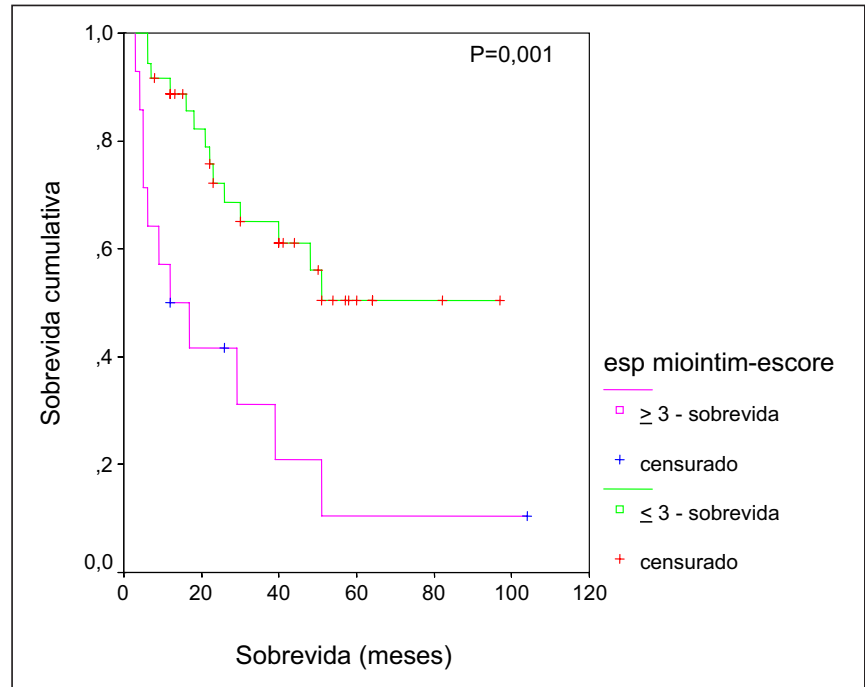

Figura 5 - Sobrevida de pacientes com FPI separados por escore de espessamento miointimal na biópsia pulmonar
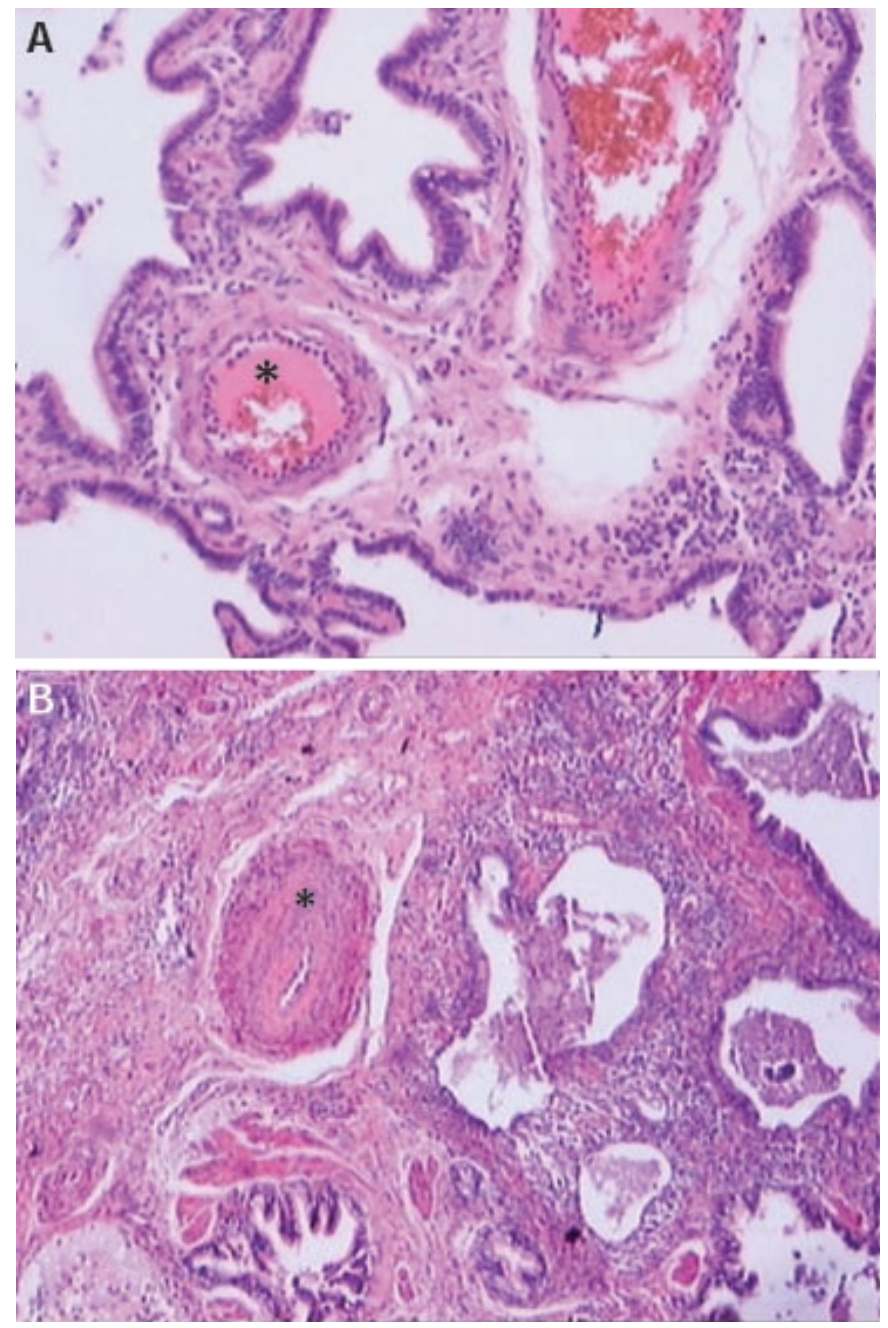

Figura 6A e B - Espessamento miointimal; $\boldsymbol{A}$ ) discreto(*); $\mathbf{B})$ acentuado $(*)$

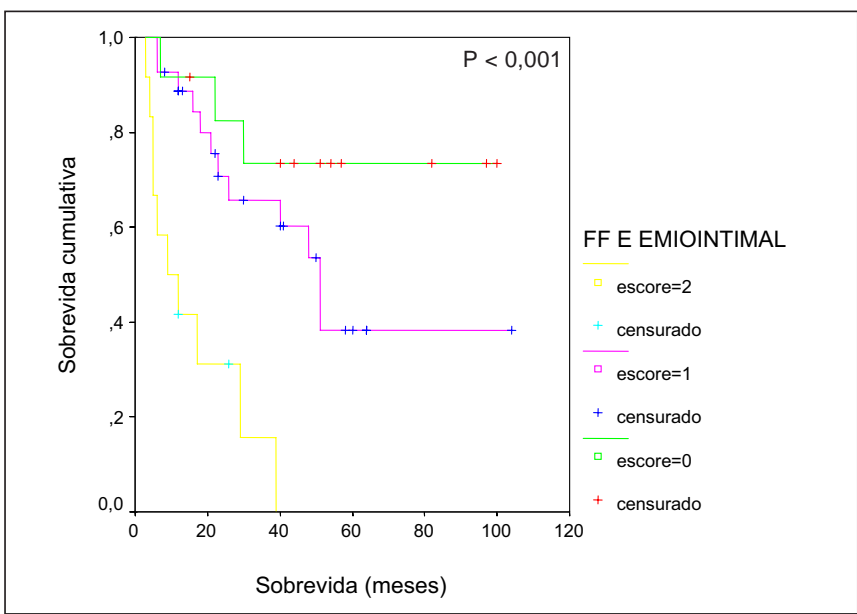

Figura 7 - Sobrevida de pacientes com FPI separados por escoresoma de focos fibroblásticos ( 0 =esparsos; 1 = profusos) e espessamento miointimal $(50 \%$ dos vasos $=0 ;>50 \%=1)$ na biópsia pulmonar 
O escore para o espessamento miointimal não se correlacionou de maneira significativa com a CVF\% (rho $=-0,18$, $p=0,24)$, mas correlacionou-se significativamente com 0 escore de faveolamento ( $r$ o $=0,38, p=0,006$ ).

Um escore de soma qualitativo simples (de 0 a 2 ) foi feito somando-se os pontos obtidos no escore de focos fibroblásticos (esparsos $=0$; demais $=1$ ) com o escore de espessamento miointimal $(<50 \%=0$; maior que $50 \%=$ 1). A sobrevida, significativamente diferente nos três grupos (log-rank $=22,01, p=0,000$ ) é mostrada na Figura 7. As medianas de sobrevida foram para o grupo com escore de $2(n=12)$ de apenas nove meses e para 0 grupo intermediário, com escore $=1(\mathrm{n}=27)$, de 51 meses. 0 grupo com escore $0(n=12)$ teve sobrevida maior que os outros dois grupos. Um escore qualitativo semelhante, envolvendo focos fibroblásticos e faveolamento, resultou em discriminação significativa dos três grupos, porém com menor separação em comparação com o escore acima citado.

Q uando os achados descritos na literatura com influência na sobrevida (CVF\%, tempo de história, sexo, tabagismo) foram analisados individualmente por regressão de Cox, apenas o tempo de história correlacionou-se de maneira inversa com a sobrevida $(p=0,023)$.

\section{DISCUSSÃO}

0 presente estudo mostra que a avaliação semiquantitativa do número de focos fibroblásticos, do faveolamento e do espessamento miointimal na biópsia pulmonar em portadores de fibrose idiopática fornece informações prognósticas relevantes.

Os fatores relacionados ao prognóstico da fibrose pulmonar idiopática foram estudados por diversos autores. As conclusões discordantes entre as séries antigas podem ser explicadas por diversos fatores, como a não uniformidade nos métodos de obtenção do material estudado, e a presença de amostras heterogêneas com inclusão de diferentes doenças pulmonares, idiopáticas ou de etiologia conhecida. A uniformidade em geral também não era preservada na avaliação histológica. Na maioria dos casos, somente celularidade e fibrose foram avaliadas, sendo que o termo celularidade foi utilizado tanto para quantificar inflamação intersticial como para avaliar o acúmulo de células no interior dos alvéolos. Em outras séries, o termo celularidade foi usado para avaliar extensão e intensidade de inflamação na parede alveolar. $\mathrm{Na}$ avaliação da fibrose, uniformidade quanto a sua graduação também não foi obedecida.

No presente estudo, a seleção dos pacientes portadores de fibrose pulmonar idiopática foi realizada de forma criteriosa. Foram excluídos pacientes com outras doenças sistêmicas, colagenoses, com uso de fármacos poten- cialmente fibrogênicos e com outras causas conhecidas que pudessem ocasionar pneumonia intersticial usual. Pacientes que morreram devido ao procedimento de biópsia pulmonar foram desconsiderados. Viés de seleção pode ser introduzido pela necessidade da biópsia para inclusão, porém, a média de idade sugere que os casos são representativos da doença. A baixa freqüência de óbitos não decorrentes de outras doenças, por outro lado, sugere que pacientes com co-morbidades significativas foram biopsiados menos freqüentemente.

0 método de obtenção do material foi uniforme: todas as biópsias foram cirúrgicas e todas foram revisadas, sendo excluídos os casos que não apresentavam quadro histológico de pneumonia intersticial usual. $\mathrm{Na}$ avaliação morfológica, os achados maiores foram semiquantificados por escore previamente publicado, ${ }^{(11)}$ utilizando-se, entretanto, os componentes individuais dos diversos achados histológicos e não os escores-soma propostos.

Casos mais antigos, em que a biópsia isolada de língula foi realizada, foram incluídos. Fragmentos de pulmão post mortem de indivíduos sem doenças intersticiais demonstram que na língula há mais fibrose e alterações vasculares em comparação com outras áreas. ${ }^{(19)} \mathrm{J}$ á em pacientes com doenças intersticiais, a biópsia de língula é geralmente representativa. ${ }^{(20)}$ Pela exclusão de casos com alterações inespecíficas ou de pulmão terminal, e pelo achado de lesões características de pneumonia intersticial usual, consideramos que as biópsias de língula eram aceitáveis.

Devido à redefinição de fibrose pulmonar idiopática, os achados do presente estudo foram comparados apenas com aqueles de estudos recentes, que incluíram 0 padrão de pneumonia intersticial usual para caracterizar a fibrose pulmonar idiopática. $(12,13,21,22)$

O sexo não influenciou a sobrevida em nosso material, achado semelhante ao observado por King et al. em 238 casos. ${ }^{(12)}$ Entretanto, as mulheres tiveram maior sobrevida no estudo de Flaherty et al.(21) Não observamos influência da idade na sobrevida. A idade correlacionou-se inversamente com a sobrevida no estudo de King et al.,(12) mas não teve influência no estudo de Flaherty et al.(21) $\mathrm{A}$ idade e o sexo não influenciaram a sobrevida no estudo de Nicholson et al., ${ }^{(22)}$ porém, apenas oito mulheres foram nele incluídas.

A maior duração dos sintomas influenciou negativamente a sobrevida nos pacientes da presente série, o que pode representar diagnóstico mais precoce na história natural da doença, melhor resposta ao tratamento na doença em fase mais inicial, ou ambos.

0 tabagismo está associado a maior risco para o desenvolvimento de fibrose pulmonar idiopática, porém, os fumantes parecem ter sobrevida maior, ${ }^{(12,21,23)} 0$ que não foi observado no presente estudo. Os fumantes apresen- 
taram maior acúmulo de macrófagos intra-alveolares como resultado do tabagismo, o que foi observado por outros autores, ${ }^{(12)}$ porém, a extensão e a intensidade deste achado não se relacionou com a sobrevida. A extensão dos demais achados histológicos não diferiu entre fumantes e não fumantes. 0 mecanismo pelo qual o tabagismo exerce influência na sobrevida de pacientes com fibrose pulmonar idiopática é desconhecido. Foi sugerido que os focos de fibrose ativa poderiam ser menos exuberantes em fumantes, ${ }^{(12)}$ porém, no presente estudo, não foram observadas diferenças entre fumantes e não fumantes no escore de focos fibroblásticos.

A o contrário de uma grande série recente, ${ }^{(23)} 0$ valor da CVF inicial não se correlacionou com a sobrevida em nosso material. A mediana de sobrevida estimada nos pacientes deste estudo foi de 48 meses. Em outras séries, a mediana de sobrevida situa-se entre 30 e 48 meses. ${ }^{(12,21,23)}$ No presente estudo, a quase totalidade de óbitos foi atribuída à fibrose pulmonar idiopática. Neste grupo etário (66 \pm 8 anos) outras causas de morte são freqüentes ${ }^{(24)} e$, portanto, a sobrevida deve ser analisada considerando-se as causas do óbito.

Em nosso estudo, a extensão dos focos fibroblásticos influenciou a sobrevida: focos esparsos associaram-se a sobrevida significativamente maior. Q uatro estudos publicados correlacionaram diversos achados de biópsia a céu aberto com a sobrevida em pacientes com fibrose pulmonar idiopática/ pneumonia intersticial usual. ${ }^{(12,13,21,22)}$ Dois desses estudos são de um mesmo centro e utilizaram material semelhante. ${ }^{(13,21)} \mathrm{Em}$ ambos, 0 escore de fibrose, como originalmente descrito por Cherniack et al., (11) correlacionou-se inversamente com a sobrevida. As biópsias foram obtidas de múltiplos locais em todos os casos. King et al. (12) observaram que a sobrevida foi mais longa em pacientes com graus menores de focos fibroblásticos. Celularidade e fibrose estabelecida não se correlacionaram com a sobrevida. A ausência completa de correlação entre o grau de fibrose estabelecida, o faveolamento e a sobrevida pode ser explicada pela seleção dos locais para biópsia: os locais de fibrose avançada foram evitados. Em nosso estudo esses locais foram incluídos e melhor correlação foi observada. Nicholson et al.(22) correlacionaram os achados de biópsia, quantificados por um novo escore, com o declínio funcional e a sobrevida em 53 pacientes com fibrose pulmonar idiopática/ pneumonia intersticial usual. Maior mortalidade e maior declínio funcional foram independentemente relacionados com escores mais elevados para os focos fibroblásticos.

U m estudo adicional avaliou, especificamente, o valor da extensão dos focos fibroblásticos, classificados por três patologistas reconhecidos, em leves, moderados e marcados. ${ }^{(14)}$ Maior escore não se associou com maior mortalidade.
A fibrose pulmonar é a via final comum de um grupo diverso de doenças pulmonares intersticiais. Existem duas vias para o desenvolvimento de fibrose pulmonar difusa: a via inflamatória, observada em quase todas as doenças pulmonares intersticiais, em que uma fase inicial de alveolite é evidente, seguida de uma fase fibrótica tardia, e a via epitelial, observada na UIP. ${ }^{(25)} \mathrm{A}$ hipótese atualmente mais aceita propõe que a FPI resulta de microlesões epiteliais com ativação das células epiteliais alveolares e reparo anormal. (26) As células epiteliais liberam fatores que induzem a migração e a proliferação de fibroblastos, e mudanças no fenótipo celular para miofibroblastos. No microambiente do pulmão lesado, os miofibroblastos induzem apoptose das células epiteliais alveolares e mudanças irreversíveis na arquitetura da matriz extracelular, resultando em um remodelamento errático do parênquima pulmonar. (25) O utros autores propuseram que a apoptose pode ser 0 evento primário na UIP. (27)

$\mathrm{Na}$ fibrose pulmonar idiopática, embora a maior parte da fibrose seja composta de feixes de colágeno acelular, "velho", pequenos agregados de miofibroblastos e fibroblastos são muitas vezes identificados. Esses agregados, chamados focos fibroblásticos, são caracterizados por células fusiformes presentes em uma matriz mixóide frou$x a$, as quais são arranjadas paralelamente ao eixo longo dos septos alveolares. Esses focos representam a organização de focos prévios de agressão e pode-se demonstrar síntese ativa de colágeno nesses locais. Eles indicam que a fibrose está ativa e, portanto, sua extensão pode representar maior ou menor probabilidade de progressão da doença. ${ }^{(3)}$ Nosso estudo corrobora a hipótese epitelial e que a extensão dos focos fibroblásticos tem valor prognóstico.

Neste estudo, comprometimento de mais de $50 \%$ dos vasos com espessamento miointimal teve alto valor preditivo para maior mortalidade na FPI. No estudo de King et al. ${ }^{12)}$ essa correlação não foi observada, o que pode ser explicado pelo fato de terem sido evitadas biópsias das áreas mais afetadas. É reconhecido que o espessamento miointimal mural na parede dos vasos é um achado comum nas áreas de fibrose. Extensa obliteração do leito vascular pulmonar irá resultar em hipertensão pulmonar, um fator reconhecido para pior prognóstico em fibrose pulmonar. (23) Um recente e provocativo estudo sugeriu que a fibrose pulmonar poderia decorrer de uma agressão primariamente vascular. ${ }^{(16)}$

Concluímos que a avaliação da extensão dos focos fibroblásticos, do grau de faveolamento e, em especial, da obliteração resultante do leito vascular pulmonar, nas biópsias pulmonares de pacientes com FPI, fornece informações prognósticas de grande interesse. 0 estudo de uma nova coorte de pacientes, já em andamento, deverá validar os achados do presente estudo. 


\section{REFERÊNCIAS}

1. Liebow AA. Definition and classification of interstitial pneumonias in human pathology. Prog Respir Res 1975;8:1-31.

2. Katzenstein ALA. Idiopathic interstitial pneumonia: classification and diagnosis. In: Churg A, Katzenstein A-LA, editors. The lung current concepts. Baltimore: Williams \& Wilkins, 1993;1-31.

3. Katzenstein ALA, Myers JL. Idiopathic pulmonary fibrosis. Clinical relevance of pathology classification. State of the art. A m J Crit Care Med 1998;157:1301-15.

4. American Thoracic Society. Idiopathic pulmonary fibrosis: diagnosis and treatment. International consensus statement. Am J Respir Crit Care Med 2000;161:646-64

5. Katzenstein ALA, Fiorelli RF. Nonspecific interstitial pneumonia/ fibrosis. $\mathrm{H}$ istologic features and clinical significance. Am J Surg Pathol 1994; 18:136-47.

6. Bjoraker JA, Ryu JH, Edwin MK, Myers J L, Tazelaar HD, Schroeder $D R$, et al. Prognostic significance of histopathologic subsets in idiopathic pulmonary fibrosis. Am J Respir Crit Care Med 1998;157:199203.

7. Cottin V, Donsbeck AV, Revel D, Loire R, Cordier J F. Nonspecific interstitial pneumonia. Individualization of a clinicopathologic entity in a series of 12 patients. Am J Respir Crit Care Med 1998;158:1286-93.

8. Travis WD, Matsui K, Moss J , Ferrans VJ . Idiopathic nonspecific interstitial pneumonia: prognostic significance of cellular and fibrosing patterns. Survival comparison with usual interstitial pneumonia and desquamative interstitial pneumonia. Am J Surg Pathol 2000;24:19-33.

9. Fukuda Y, Basset F, Ferrans VJ, Yamanaka N. Significance of early intraalveolar fibrotic lesions and integrin expression in lung biopsy specimens from patients with idiopathic pulmonary fibrosis. Hum Pathol 1995;26:53-61.

10. Myers J L, Katzenstein ALA. Epitelial necrosis and alveolar collapse in the pathogenesis of usual interstitial pneumonia. Chest 1988;94:1309-11.

11. Cherniack RM, Colby TV, Flint A, Thurlbeck WM, Waldron J, Ackerson $L$, et al. Quantitative assessment of lung pathology in idiopathic fibrosis. Am J Respir Dis 1991;144:892-900.

12. King TE J r, Schwarz MI, Brown K, Tooze J A, Colby TV, Waldron J A $\mathrm{J} r$, et al. Idiopathic pulmonary fibrosis: relationship between histopathologic features and mortality. Am J Respir Crit Care Med 2001;164: 1025-32.

13. Gay SE, Kazerooni EA, Toews GB, Lynch J P 3rd, Gross BH, Cascade $P N$, et al. Idiopathic pulmonary fibrosis. Predicting response to therapy and survival. Am J Respir Crit Care Med 1998;157:1063-72.
14. Flaherty KR, Colby TV, Travis WD, Towes GB, Flint A, et al. Prognostic value of fibroblastic foci in patients with usual interstitial pneumonia. Chest 2001;120(Suppl):76-7.

15. Strieter RM, Belperio J A, Keane MP. CXC chemokines in angiogenesis related to pulmonary fibrosis. Chest 2002;120(Suppl 1):298-301.

16. Magro CM, Allen J, Pope-Harman A, Waldman J, Moh P, Rothrauff $\mathrm{S}$, et al. The role of microvascular injury in the evolution of idiopathic pulmonary fibrosis. Am J Clin Pathol 2003;119:556-67.

17. Pereira CAC, Barreto SP, Simões J G, Pereira FWL, Gerstler J G, Nakatani J. Valores de referência para espirometria em uma amostra da população brasileira adulta. J Pneumol 1992;18:10-22.

18. Ferreira RG, Coletta ENAM, Giannotti Filho 0 . Avaliação de parâmetros histológicos na pneumonia intersticial usual (fibrose pulmonar idiopática). J Pneumol 2000;26:279-85.

19. Newman SL, Michel RP, Wang NS. Lingular lung biopsy: is it representative? Am Rev Respir Dis 1985;132:1084-6.

20. Flint A, Martinez FJ, Young ML, Whyte RI, Toews GB, Lynch J P 3rd. Influence of sample number and biopsy site on the histologic diagnosis of diffuse lung disease. Ann Thorac Surg 1995;60:1605-7.

21. Flaherty KR, Toews GB, Travis WD, Colby TV, Kazerooni EA, Gross $\mathrm{BH}$, et al. Clinical significance of histological classification of idiopathic interstitial pneumonia. Eur Respir J 2002;19:275-83.

22. Nicholson AG, Fulford LG, Colby TV, Du Bois RM, Hansell DM, Wells $A U$. The relationship between individual histologic features and disease progression in idiopathic pulmonary fibrosis. Am J Respir Crit Care Med 2002;166:173-7.

23. King TE J r, Tooze JA, Schwarz MI, Brown KR, Cherniack RM. Predicting survival in idiopathic pulmonary fibrosis: scoring system and survival model. Am J Respir Crit Care Med 2001;164:1171-81.

24. Panos RJ, Mortenson RL, Niccoli SA, King TE J r. Clinical deterioration in patients with idiopathic pulmonary fibrosis: causes and assessment. Am J Med 1990;88:396-404.

25. Pardo A, Selman M. Molecular mechanisms of pulmonary fibrosis. Front Biosci 2002;7:1743-61.

26. Selman M, King TE, Pardo A. Idiopathic pulmonary fibrosis: prevailing and evolving hypotheses about its pathogenesis and implications for therapy. Ann Intern Med 2001;134:136-51.

27. Barbas-Filho JV, Ferreira MA, Sesso A, Kairalla RA, Carvalho CR, Capelozzi VL. Evidence of type II pneumocyte apoptosis in the pathogenesis of idiopathic pulmonary fibrosis (IFP)/ usual interstitial pneumonia (UIP). J Clin Pathol 2001;54:132-8. 